Pietro Cicuta

Dominic Vella 



\title{
Wrinkling in the deflation of elastic bubbles
}

\author{
Elodie Aumaitre ${ }^{1}$, Sebastian Knoche ${ }^{2}$, Pietro Cicuta ${ }^{1}$ and Dominic Vella ${ }^{3,4}$ \\ 1 Cavendish Laboratory, University of Cambridge, JJ Thomson Avenue, CB3 0HE Cambridge, UK \\ 2 Faculty of Physics, Technische Universität Dortmund, 44221 Dortmund, Germany \\ 3 OCCAM, Mathematical Institute, 24-29 St Giles', Oxford, OX1 3LB, UK \\ 4 Department of Applied Mathematics and Theoretical Physics, University of Cambridge, Wilberforce Road, \\ Cambridge, CBз OWA, UK
}

\author{
PACS $46.32 .+\mathrm{x}-$ Static buckling and instability \\ PACS 68.03.-g-Interfaces gas-liquid \\ PACS 62.20.Dc - Elasticity, elastic constants
}

\begin{abstract}
The protein hydrophobin HFBII self-assembles into very elastic films at the surface of water; these films wrinkle readily upon compression. We demonstrate and study this wrinkling instability in the context of non-planar interfaces by forming HFBII layers at the surface of bubbles; the interfaces are then compressed by deflating the bubble. By varying the initial concentration of the hydrophobin solutions, we are able to show that buckling occurs at a critical packing fraction of protein molecules on the surface. Independent experiments show that at this packing fraction the interface has a finite positive surface tension, and not zero surface tension as is usually assumed at buckling. We attribute this non-zero wrinkling tension to the finite elasticity of these interfaces. We develop a simple geometrical model for the evolution of the wrinkle length with further deflation, and show that wrinkles start close to the needle used for deflation and grow rapidly towards the mid-plane of the bubble. This geometrical model yields predictions for the length of wrinkles in good agreement with experiments, independently of the rheological properties of the adsorbed layer.
\end{abstract}

Introduction. - From the formation of finger prints [1] to geological structures [2], the buckling of thin sheets in various situations can be explained by the onset of an elastic instability in which an in-plane compression leads to an out-of-plane deformation. The theory of elasticity of a beam or a plate [3] can be used to predict the wavelength and amplitude of the wrinkles by balancing the bending and stretching energies $[4,5]$. Conversely, buckling instabilities have frequently been used to infer properties of materials, such as Young's modulus and thickness, based on measurements of the wrinkling pattern $[6,7]$.

Wrinkling is also observed in materials with more complicated rheologies than simple Hookean solids. For example, wrinkling has been observed in surfactant and protein monolayers [8] as well as in 'particle rafts' $[9,10]$. While these systems have been well studied, theoretical models are often based on the assumption that buckling occurs when the interfacial tension vanishes (so that deformation out of the plane costs little or no surface energy). This hypothesis has been verified experimentally for films with no significant elastic shear modulus, such as lung surfac- tants [8]. However, the literature provides many examples of layers buckling before this zero tension state is reached. For example, interfaces coated with spherical [11] and ellipsoidal [12] colloidal particles buckle upon compression whilst the interfacial tension is still a significant fraction of its value before coating. Although commonly observed, the buckling of layers with finite shear modulus has yet to be fully understood from a theoretical point of view. Various models based on the mechanics of elastic sheets have been proposed to explain the observed wavelength of wrinkles $[9,13]$. It is also observed that folds may form in such systems; one proposal is that the formation of these folds is analogous to the formation of Griffith cracks in solid plates [14]. Nevertheless, no existing model is able to explain the observation of buckling at finite surface tension and, in particular the sensitivity of the buckling tension on the geometry of the experimental apparatus $[10,15]$.

The observation that the onset of wrinkling has a vital geometrical aspect makes it surprising that most studies of the buckling and collapse of interfacial monolayers focus largely on compression in planar geometries such as a 
Langmuir trough. Recently, Stanimirova et al. [16] have observed wrinkles on a pendant droplet covered by the surfactant Saponin, which has the potential to develop highly elastic monolayers. Here, we develop a controlled setup to investigate the wrinkling of hydrophobin HFBII films on the surface of a bubble where the compression of the interface is achieved by deflation of the bubble. Our use of the protein hydrophobin HFBII is motivated by recent interest in its ability to self-assemble into highly elastic films. This high level of elasticity is believed to explain HFBII's capacity to stabilize foams on long timescales [17], which in turn makes it of interest in a range of food and medical applications. We focus on studying the wrinkling of hydrophobin films in this geometry since it is both one of practical interest and because it removes some of the complexities that arise studying wrinkling in planar Langmuir troughs, such as friction with side walls [15]. We show that buckling appears to occur when the hydrophobin layer reaches a critical surface concentration but also demonstrate that some features of the problem, such as the rapid rate at which wrinkles grow, can be understood using purely geometrical ideas.

Experiment. - Class II hydrophobin (HFBII) from Trichoderma Reesei was a gift from Unilever Global Development Centre and was obtained from VTT Biotechnology (Espoo, Finland). Details of the preparation are described elsewhere [18,19]. A drop tensiometer (First Ten Angstroms, UK) comprising of a precise microstage and a camera was used for the buckling experiment. A bubble was formed at the tip of a J-needle dipped into a cuvette filled with a hydrophobin solution, left to equilibrate for 20 minutes (to reach the equilibrium surface tension of the surrounding solution), and then deflated by sucking the air back into the syringe gently, see Fig. 1. The volume and surface area at which the bubble wrinkles can be recorded as well as the length of the wrinkles. The J-needles with outer diameters of $0.75 \mathrm{~mm}$ and $1.25 \mathrm{~mm}$ were made by bending blunt stainless steel needles with round pliers. The experiment was repeated varying the initial volume of the bubble (typically between 2 and $10 \mu \mathrm{L}$ ) and the surface tension of the bulk hydrophobin solution. The equilibrium surface tension of the hydrophobin solutions was measured by Wilhelmy plate tensiometry (process tensiometer K12, Kruss GmbH, Hamburg).

Results. - As the bubble is deflated we see that wrinkles appear first at a critical value of the bubble surface area $S_{\text {wrink }}$ (see Fig. 2a-c). Experiments show that this critical value is proportional to the surface area of the bubble before deflation, $S_{e q}$, for bubbles of different initial sizes (see Fig. 2a-c). We note that the compression ratio at wrinkling, $S_{\text {wrink }} / S_{e q}$, varies with the initial equilibrium surface tension of the hydrophobin solution. As might be expected, $S_{\text {wrink }} / S_{e q} \rightarrow 1$ as the concentration of hydrophobin in the bulk solution (and hence on the interface) increases; the bubble wrinkles with a smaller amount of compression. This observation suggests that
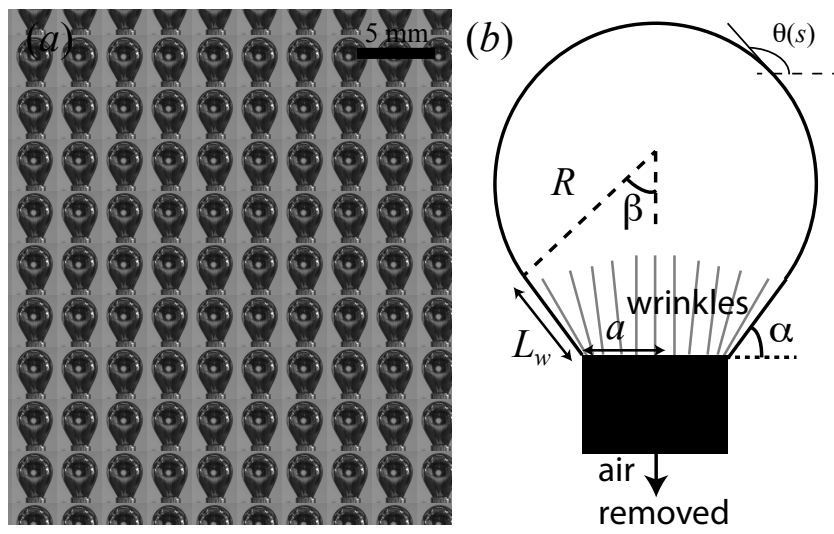

Fig. 1: (a) Experimental image of a wrinkled bubble in hydrophobin solution. The bubble is initially allowed to equilibrate with the bulk solution before it is deflated causing the wrinkling seen here. (b) Schematic diagram illustrating the model parameters used here, namely the radius of the needle to which the bubble is attached, $a$, the typical radius of curvature of the bubble, $R$, and the length of the wrinkled portion $L_{w}$.

wrinkles form when the molecules reach a critical packing fraction. This hypothesis may be confirmed by estimating the molecular area at which wrinkling first occurs. To do this, we use a plot of the surface pressure, $\Pi$, (defined in terms of the surface tensions of the clean and dirty interfaces, $\gamma_{c}$ and $\gamma_{d}$, respectively, by $\Pi=\gamma_{c}-\gamma_{d}$ ) as a function of the average area occupied per molecule, $A_{m}$. Fig. 2 shows such a surface pressure isotherm for an interface compressed in a Langmuir trough ${ }^{1}$. The equilibrium surface tension of the hydrophobin solution, together with the surface pressure isotherm, gives an estimate of the average surface area per molecule on the surface of the bubble initially, $A_{i}^{m}$. The average surface area per molecule at the onset of wrinkling, $A_{w}^{m}=A_{i}^{m} \times S_{w r i n k} / S_{e q}$, can then be calculated and the surface pressure at wrinkling $\Pi_{w}=\Pi\left(A_{w}^{m}\right)$ deduced from the isotherm. The results of this procedure are shown in Fig. 2 and demonstrate that the value of $\Pi_{w}$ is well-defined and is, to within experimental errors, independent of the equilibrium surface pressure of the solution. Here, we observe that the apparent surface tension at wrinkling is not negligible $\left(\gamma_{\text {wrink }}=\gamma_{C}-\Pi \simeq 72.8-30 \simeq 42.8 \mathrm{mN} / \mathrm{m}\right)$. We note that this estimate is based on the surface-averaged area per molecule at wrinkling. Since wrinkling cannot occur unless the stress within the interface is locally compressive, we conclude that the true stress state within the layer must be inhomogeneous and anisotropic. However, it is not possible to distinguish between the contributions to this stress state from the elastic response of the interface and variations in the 'true' surface tension [20]; we

${ }^{1}$ It should be emphasised here that, as studied in previous work [15], the measurements of surface pressure returned by a Wilhelmy plate in a rigid layer might not correspond simply to the pure surface pressure. However, this technique can still be useful to provide an idea of the surface area per molecule and pressure at wrinkling. 
therefore refer to the stress state of the layer in what follows. Finally, we note that the value of $\gamma_{\text {wrink }}$ measured here is consistent with observations by Kisko et al. [21] on hydrophobin HFBII films, who imaged the collapse of the layer by Brewster Angle Microscopy at a surface pressure $\geq 30 \mathrm{mN} / \mathrm{m}$.

Once wrinkles have appeared near the needle, their length grows dramatically as the bubble is deflated further. This is illustrated in Fig. 3, which shows the wrinkle length as a function of bubble volume for a range of initial bubble volumes and bulk solution concentrations. In the next section we present a minimal model aimed at explaining this behaviour. Our model is based on the assumption that the surface pressure is not isotropic within the thin layer, as is demonstrated by the formation of orientated wrinkles. Furthermore, we assume that wrinkling occurs "far from threshold" [22], so that the stress along the wrinkles dominates that perpendicular to the wrinkles. Because of the anisotropy of the stress state, the shape of the bubble will deviate significantly from the LaplaceYoung equation, as discussed by Stanimirova et al. [16]. We therefore begin by discussing the equations describing the shape of such a bubble.

Model. - We model the bubble as an elastic capsule of thickness $h$ and Young's modulus $E$. Initially the bubble has some typical radius, $R$, and is subject to an internal pressure $p \approx 2 \gamma_{e} / R$ with $\gamma_{e}$ being the equilibrium surface tension coefficient for the hydrophobin solution. Studies of the deformation of a pressurized spherical capsule $[23,24]$ show that the bending stiffness of the capsule may be neglected if the dimensionless tension in the capsule due to the internal pressure

$$
\tau=\frac{p}{E}\left(\frac{R}{h}\right)^{2} \gg 1 .
$$

For the hydrophobin-coated bubbles studied here with a thickness $h \simeq 2 \mathrm{~nm}$, the effective Young's modulus $E$ may be estimated from the surface elastic modulus $\varepsilon=E h$ where $\varepsilon$ can in turn be estimated from the slope of compression isotherms as $\varepsilon=A \times(d \Pi / d A)$. For $\gamma_{e} \simeq 50 \mathrm{mN} / \mathrm{m}, \varepsilon \simeq 30 \mathrm{mN} / \mathrm{m}$ and $E \simeq 15 \mathrm{MPa}$. With $R \approx 1 \mathrm{~mm}$, we therefore find that $\tau \gtrsim 10^{5}$, i.e. $\tau \gg 1$ : the bending stiffness may safely be neglected in what follows.

The stresses within the surface, the meridional stress $\sigma_{s}$ and the azimuthal, or hoop, stress $\sigma_{\phi}$, determine the shape of the capsule according to the normal and tangential force balance equations

$$
\frac{1}{r} \frac{\mathrm{d}}{\mathrm{d} s}\left(r \sigma_{s}\right)-\frac{\cos \theta}{r} \sigma_{\phi}=0
$$

and

$$
p=\sigma_{s} \frac{\mathrm{d} \theta}{\mathrm{d} s}+\sigma_{\phi} \frac{\sin \theta}{r}-\rho g z .
$$

These equations are also known as the equations of membrane-shell theory $[25,26]$. In eqns. (2)-(3), $s$ is the arc length measured from the base of the bubble (where it contacts the needle) along the bubble surface and $\theta(s)$ is the inclination of the surface tangent to the horizontal. The position of a point on the bubble surface, $[r(s), z(s)]$, is determined in terms of the arc length $s$ and interfacial inclination $\theta(s)$ by the geometrical relationships

$$
\frac{\mathrm{d} r}{\mathrm{~d} s}=\cos \theta, \quad \frac{\mathrm{d} z}{\mathrm{~d} s}=\sin \theta
$$

Equations (2), (3) and (4) are to be solved together with the boundary conditions that

$$
z(0)=0, \quad r(0)=a, \quad r\left(s_{\max }\right)=0, \quad \theta\left(s_{\max }\right)=\pi
$$

for $0 \leq s \leq s_{\max }$ for some $s_{\max }$. In reality, the value of $s_{\max }$ will be determined by the volume of the bubble $V$ according to

$$
V=\int_{0}^{z\left(s_{\max }\right)} \pi r^{2} \mathrm{~d} z=\int_{0}^{s_{\max }} \pi r^{2} \sin \theta \mathrm{d} s .
$$

We note that upon letting $\sigma_{s}=\sigma_{\phi}=\gamma$ (as is usually the case for bubbles) then eq. (3) becomes the Laplace-Young equation [27], and eq. (2) is identically satisfied because of the geometrical relationship of eq. (4).

We intend to study the numerical solutions of eqns. (2)(3) in the future. This requires the assumption of a constitutive relationship between stresses and strains. However, to obtain some physical understanding of the growth of wrinkles, we present here a simplified, geometrical model. This approach eliminates some of the problems and uncertainties associated with our incomplete knowledge of the mechanical behaviour, i.e. the rheology, of hydrophobin monolayers.

In studies of the wrinkling of membranes (sheets with negligible bending stiffness), it is common to assume that the stress in the direction perpendicular to the wrinkles is significantly smaller than that in the direction along the wrinkles [5, 22, 28]; wrinkling relieves the compressive stress. For the wrinkled bubbles studied here, therefore, we assume that $\sigma_{\phi} \ll \sigma_{s}$ within the wrinkled region. Furthermore, we assume that $\sigma_{s} / R \gg p+\rho g z\left(s_{\max }\right)$ so that eq. (3) becomes

$$
\sigma_{s} \frac{\mathrm{d} \theta}{\mathrm{d} s} \approx 0
$$

within the wrinkled region $0 \leq s \leq L_{w}$ with $L_{w}$ denoting the length of the wrinkles. This approximation is motivated by the experimental observation that within the wrinkled region of the bubbles $\theta \approx \alpha$, a constant. In the unwrinkled region, we assume that the stress state is isotropic so that $\sigma_{\phi}=\sigma_{s}$ and, for simplicity, we take $\sigma_{\phi}=\sigma_{s}=\sigma_{0}$ with the additional assumption that $p \gg \rho g z\left(s_{\max }\right)$ so that the unwrinkled portion of the bubble is simply a spherical cap, again as is observed experimentally.

Taken together our assumptions model the wrinkled bubble as consisting of a wrinkled part, in which the interface makes a constant angle $\alpha$ with the horizontal, connected to an unwrinkled, spherical cap. We note that 


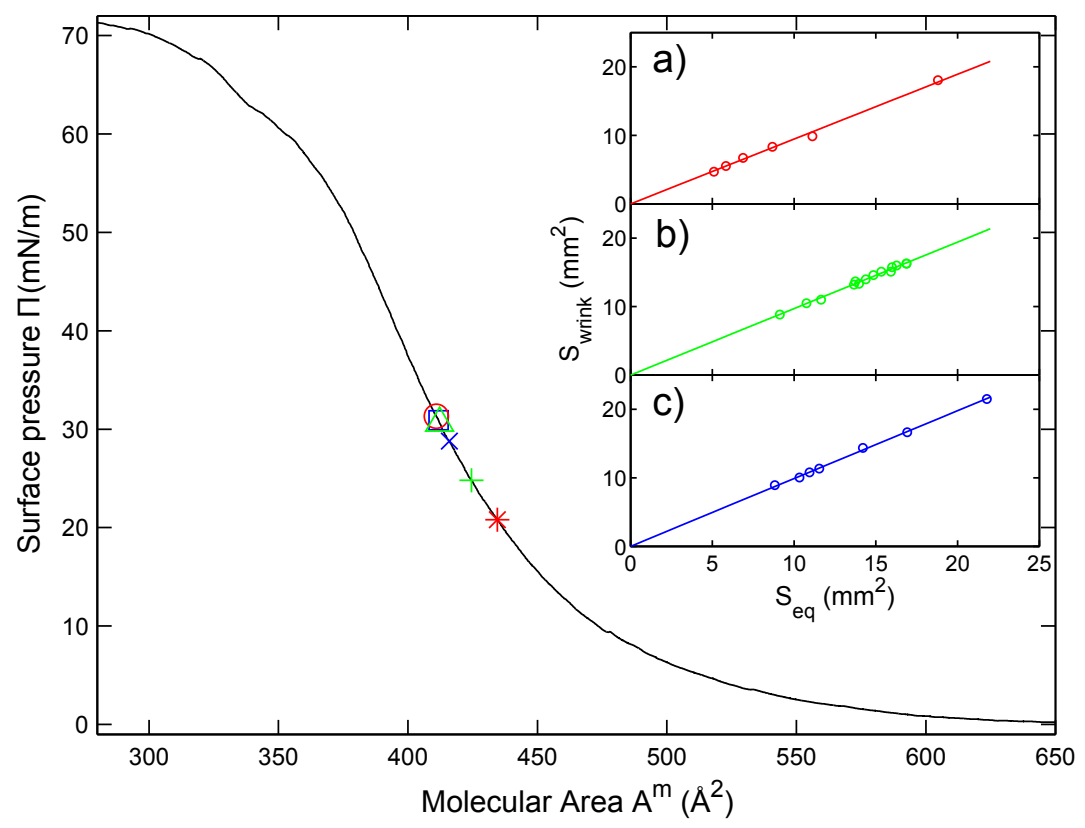

Fig. 2: There is strong evidence that buckling occurs at a fixed area per molecule. The inset illustrates that the surface area of the bubble when the first wrinkles appear, $S_{\text {wrink }}$, is proportional to the surface area of the bubble before compression, $S_{\text {eq }}$. Each marker represents a new bubble, and a single compression experiment. The data sets in (a), (b) and (c) correspond to three hydrophobin solutions respectively with surface tensions of $52 \mathrm{mN} / \mathrm{m}, 48 \mathrm{mN} / \mathrm{m}$ and $44 \mathrm{mN} / \mathrm{m}$. These initial surface pressures are reported on an isotherm of compression $(*,+, \times)$ obtained from compression of a hydrophobin layer in a Langmuir trough, as shown in the main panel. The compression ratio $S_{\text {wrink }} / S_{e q}$ can be deduced from the inset plots, and used to calculate the mean molecular area and surface pressure at wrinkling $(\circ, \triangle, \square)$; all three markers coincide.

with this assumption, elementary geometry ensures that $\alpha=\beta$, with $\alpha$ and $\beta$ defined in Figure 1. The advantage of these simplifications are two-fold: firstly this model is analytically tractable, secondly we neglect the details of the stress state within the bubble, which depends on both the detailed rheology of the interface as well as the history of bubble deformations from an initial condition [26]. To close the model, we assume that the surface area of the bubble is fixed at its value at the onset of wrinkling, $S=S_{\text {wrink }}$; this corresponds to assuming that the molecules are already at maximum packing and cannot pack further.

The connection between the wrinkled and unwrinkled parts occurs at an unknown arclength $s=L_{w}$. The position of the boundary of the wrinkled region, $L_{w}$ must be determined as part of the solution of the problem. Indeed, the position of the wrinkle extent has recently been a focus of considerable theoretical and experimental effort in related systems $[7,20,22,23]$. We therefore focus on understanding the evolution of the wrinkle length as the volume of the bubble is decreased further beyond the onset of wrinkling.

The surface area of the bubble is the sum of that of the section of a cone (the wrinkled region) and a spherical cap of radius $R$ (the unwrinkled region), which may be expressed as

$$
\tilde{S}=\frac{S}{a^{2}}=\frac{\pi}{\cos \alpha}\left[\frac{R^{2}}{a^{2}}(1+\cos \alpha)^{2}-1\right]
$$

while the volume is

$$
\tilde{V}=\frac{V}{a^{3}}=\frac{\pi}{3}\left[\frac{R^{3}}{a^{3}} \frac{(1+\cos \alpha)^{2}}{\cos \alpha}-\tan \alpha\right] .
$$

Here we are using the radius of the needle, $a$, to nondimensionalize all lengths since this is the only length scale remaining in the problem after the capillary length has been eliminated by neglecting the effect of gravity. $\tilde{S}$ and $\tilde{V}$ are thus the dimensionless surface area and volume of the bubble, respectively. Geometry may also be used to show that the dimensionless length of the wrinkles is given by

$$
\tilde{L}_{w}=\frac{L_{w}}{a}=\frac{R}{a} \tan \alpha-\sec \alpha .
$$

For a given initial surface area at the wrinkling point $\tilde{S}=\tilde{S}_{w}$, we may use the angle $\alpha$ to parametrize the bubble shapes with this $\tilde{S}$. To do this, we calculate the bubble radius $R\left(\tilde{S}_{w} ; \alpha\right)$ from eq. (8) and use this value in eqns. (9) and (10) to give the corresponding bubble volume and wrinkle length. In this way, it is possible to plot the wrinkle length as a function of bubble volume for different initial surface areas. Such a plot is shown in Fig. 3 for a variety of different values of $\tilde{S}_{w}$. We note that the agreement between theoretical predictions for the length of the wrinkles and experimental observations is extremely good; we emphasize especially that there are no fitting parameters in this model.

The most striking feature of the theoretical and experimental curves in Fig. 3 is that the curves are normal to 


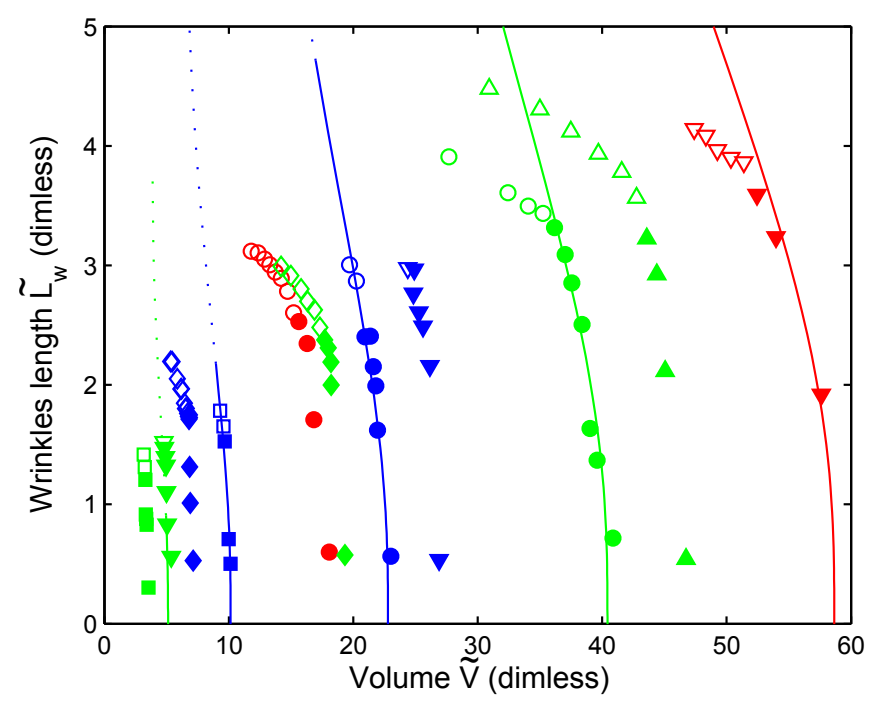

Fig. 3: Wrinkles grow very quickly under compression, and the length is well described by a simple geometrical model. The dimensionless wrinkle length is plotted as a function of bubble volume. Each set of data is a different bubble. The experimental results (markers) are shown for different hydrophobin solution surface tensions (red: $52 \mathrm{mN}$, blue: $48 \mathrm{mN} / \mathrm{m}$, green: $44 \mathrm{mN} / \mathrm{m}$ ). Lengths are non-dimensionalized by the radius of the needle, $a$, and volumes by $a^{3}$. Solid lines show the theoretical predictions from the minimal geometrical model presented in the text, with no fitting parameters. The growth of the wrinkles beyond the mid-plane of the bubble is indicated by open markers (experiments) and dashed curves (theory); the differences here are due to the simplification in the model, which assumes a straight wrinkled region.

the $x$-axis at the point where they intersect it: the wrinkle length grows continuously (cf. a second order phase transition) rather than discontinuously. This result may be demonstrated analytically by showing that $V^{\prime}\left(\alpha_{0}\right)=0$ where $\alpha_{0}$ is such that $L_{w}\left(\alpha_{0}\right)=0$, which in turn requires that $R\left(\alpha_{0}\right) \sin \alpha_{0}=a$.

Conclusion. - We have studied the deflation induced buckling of bubbles coated by hydrophobin. This is novel both in the sense that wrinkled bubbles may be of interest in applications and also because it may enable us in future to study the rheology of such interfaces in more realistic, curved geometries than the use of a Langmuir trough currently allows. This latter feature is particularly important since numerous studies have shown that the use of a Langmuir trough and Wilhelmy plate introduce geometrical complications that may invalidate results for practically relevant scenarios.

Our study has shown that the wrinkling instability first occurs when the molecules reach a critical packing fraction. The presence of elastic effects within the interface means that the surface tension of the interface does not vanish at this packing fraction. Furthermore, the geometrical nature of the wrinkling instability was highlighted by a simple geometrical model using a minimal set of physi- cally based assumptions that was able to predict the evolution of the length of wrinkles post buckling.

\section{$* * *$}

EPSRC and Unilever plc. have funded this project through a CASE award. This publication is based on work supported in part by Grant No. KUK-C1-013-04, made by King Abdullah University of Science and Technology (KAUST) and in part by an Oppenheimer Early Career Research Fellowship (D.V.).

\section{REFERENCES}

[1] Kucken M. and Newell A. C., Europhys. Lett. , 68 (2004) 141.

[2] Hudleston P. J. and Lan L., J. Struct. Geol. , 15 (1993) 253 .

[3] Landau L., Lifshitz E., Kosevich A., Pitaevski L., Sykes J. and ReID W., Theory of Elasticity (Butterworth-Heinemann) 1986.

[4] Milner S., Joanny J. and Pincus P., Europhys. Lett. , 9 (1989) 495.

[5] Cerda E. and Mahadevan L., Phys. Rev. Lett., 90 (2003) 074302.

[6] Stafford C. M., Harrison C., Beers K. L., Karim A., Amis E. J., VanLandingham M. R., Kim H.-C., Volksen W., Miller R. D. and Simonyi E. E., Nature Mat. , 3 (2004) 545.

[7] Huang J., Juszkiewicz M., de Jeu W. H., Cerda E., Emrick T., Menon N. and Russell T. P., Science, 317 (2007) 650.

[8] Pocivavsek L., Frey S. L., Krishan K., Gavrilov K., Ruchala P., Waring A. J., Walther F. J., Dennin M., Witten T. A. and Lee K. Y. C., Soft Matter, 4 (2008) 2019.

[9] Vella D., Aussillous P. and Mahadevan L., Europhys. Lett., 68 (2004) 212.

[10] Cicuta P. and Vella D., Phys. Rev. Lett. , 102 (2009) 138302.

[11] Zang D., Stocco A., Langevin D., Wei B. and Binks B. P., Phys. Chem. Chem. Phys., 11 (2009) 9522.

[12] Basavaraj M. G., Fuller G. G., Fransaer J. and VERMANT J., Langmuir, 22 (2006) 6605.

[13] Zhang Q. and Witten T. A., Phys. Rev. E , 76 (2007) 041608.

[14] Lu W., Knobler C. M., Bruinsma R. F., Twardos M. and Dennin M., Phys. Rev. Lett. , 89 (2002) 146107.

[15] Aumaitre E., Vella D. and Cicuta P., Soft Matter, 7 (2011) 2530.

[16] Stanimirova R., Marinova K., Tcholakova S., Denkov N. D., Stoyanov S. and Pelan E., Langmuir 27 (2011) 12486.

[17] Cox A. R., Cagnol F., Russell A. B. and Izzard M. J., Langmuir, 23 (2007) 7995.

[18] Bailey M. J., Askolin S., Horhammer N., Tenkanen M., Linder M., Penttila M. and Nakari-Setala T., Appl. Microbio. Biotech., 58 (2002) 721.

[19] Linder M., Selber K., Nakari-Setala T., Qiao M., Kula M.-R. and Penttila M., Biomacromolecules , 2 (2001) 511. 
[20] Vella D., Adda-Bedia M. and Cerda E., Soft Matter , 6 (2010) 5778.

[21] Kisko K., Szilvay G. R., Vuorimaa E., LemmetyiNen H., Linder M. B., Torkkeli M. and SerimaA R., Langmuir, 25 (2009) 1612.

[22] Davidovitch B., Schroll R. D., Vella D., AddaBedia M. and Cerda E., Proc. Natl. Acad. Sci. , 108 (2011) 18227.

[23] Vella D., Ajdari A., Vaziri A. and Boudaoud A., Phys. Rev. Lett., 107 (2011) 174301.

[24] Vella D., Ajdari A., Vaziri A. and Boudaoud A., J. R. Soc. Interface, 9 (2012) 448.

[25] Timoshenko S. and Woinowsky-Krieger S., Theory of Plates and Shells (McGraw-Hill) 1959.

[26] KNoche S., Deformation and buckling of elastic capsules Master's thesis Technische Universitat Dortmund (2011).

[27] Ravera F., Liggieri L. and Loglio G., Interfacial Rheology (Brill) 2009 Ch. 5 p. 148.

[28] Stein M. and Hedgepeth J. M., Tech. Rep. NASA, (1961) . 



\section{RECENT REPORTS}

12/44 The 'Sticky Elastica': Delamination blisters beyond small deformations

Wagner

Vella

12/45 Stochastic models of intracellular transport $\quad$ Bressloff

Newby

12/46 The effects of noise on binocular rivalry waves: a stochastic neu- Webber ral field model

Bressloff

12/47 An Ensemble Bayesian Filter for State Estimation Farmer

12/48 Simulation of cell movement through evolving environment: a fic- Séguis titious domain approach

Burrage

Erban

Kay

12/49 The Mathematics of Liquid Crystals: Analysis, Computation and

Majumdar Applications

12/50 Fourier spectral methods for fractional-in-space reaction-diffusion equations

Bueno-Orovio

Kay

Burrage

12/51 Meniscal tear film fluid dynamics near Marx's line

Zubkov

Breward

Gaffney

12/52 Validity of the Cauchy-Born rule applied to discrete cellular-scale models of biological tissues

Davit

Osborne

Byrne

Gavaghan

Pitt-Francis

12/53 A thin rivulet or ridge subject to a uniform transverse shear stress at its free surface due to an external airflow

Sullivan

Paterson

Wilson

Duffy

12/54 The Stokes boundary layer for a thixotropic or antithixotropic fluid

McArdle

Pritchard

Wilson

12/55 Thermoviscous Coating and Rimming Flow

Leslie

Wilson

Duffy

$12 / 56$ On the anomalous dynamics of capillary rise in porous media

Shikhmurzaev Sprittles

12/57 Compactly supported radial basis functions: how and why? Zhu

12/58 Multiscale reaction-diffusion algorithms: pde-assisted Brownian Franz dynamics 
12/59 Numerical simulation of shear and the Poynting effects by the fi- Mihai nite element method: An application of the generalised empirical Goriely inequalities in non-linear elasticity

12/60 From Brownian dynamics to Markov chain: an ion channel exam- Chen ple

Erban

Chapman

12/61 Three-dimensional coating and rimming flow: a ring of fluid on a

Leslie rotating horizontal cylinder

Wilson

Duffy

12/62 A two-pressure model for slightly compressible single phase flow in bi-structured porous media

Soulaine

Davit

Quintard

12/63 Mathematical modelling plant signalling networks

Muraro

Byrne

King

Bennett

12/64 A model for one-dimensional morphoelasticity and its application

Menon to fibroblast-populated collagen lattices

Hall

McCue

McElwain

12/65 Effective order strong stability preserving RungeKutta methods

Hadjimichael

Macdonald

Ketcheson

Verner

12/66 Morphoelastic Rods Part I: A Single Growing Elastic Rod

Moulton

Lessinnes

Goriely

Copies of these, and any other OCCAM reports can be obtained from:

Oxford Centre for Collaborative Applied Mathematics

Mathematical Institute

24 - 29 St Giles'

Oxford

OX1 3LB

England

www.maths.ox.ac.uk/occam 